\title{
Asymptotic behavior of a nonlinear hyperbolic heat equation with memory*
}

\author{
Claudio GIORGI, Vittorino PATA \\ Dipartimento di Matematica \\ Università di Brescia \\ I-25123 Brescia, Italy \\ e-mail: giorgi@ing.unibs.it; pata@ing.unibs.it
}

\begin{abstract}
In this paper we investigate the asymptotic behavior, as time tends to infinity, of the solutions of an integro-differential equation describing the heat flow in a rigid heat conductor with memory. This model arises matching the energy balance, in presence of a nonlinear time-dependent heat source, with a linearized heat flux law of the Gurtin-Pipkin type. Existence and uniqueness of solutions for the corresponding semilinear system (subject to initial history and Dirichlet boundary conditions) is provided. Moreover, under proper assumptions on the heat flux memory kernel and the magnitude of nonlinearity, the existence of a uniform absorbing set is achieved.
\end{abstract}

2000 Mathematics Subject Classifications: 35B40, 35L70, 45K05.

Key words: Hyperbolic heat equation, memory kernel, existence and uniqueness, uniform absorbing set.

\section{Introduction}

We consider the following semilinear integro-differential equation in a bounded domain $\Omega \subset \mathbb{R}^{3}$ :

$$
\begin{aligned}
& u_{t}(x, t)-\int_{0}^{\infty} k(\sigma) \Delta u(x, t-\sigma) d \sigma+g(u(x, t))=f(x, t) \quad \text { in } \Omega \times \mathbb{R}^{+} \\
& u(x, t)=0 \quad x \in \partial \Omega \quad t \in \mathbb{R} \\
& u(x, t)=u_{0}(x, t) \quad x \in \Omega \quad t \leq 0
\end{aligned}
$$

where $k$ is a positive kernel decreasing to zero, whose properties will be specified later.

*This work has been partially supported by the Italian MURST '98 Research Projects "Mathematical Models for Materials Science" and "Problems and Methods in the Theory of Hyperbolic Equations". 
Problem (1.1) models temperature evolution of a rigid, isotropic, homogeneous heat conductor with linear memory, which occupies a fixed domain $\Omega \subset \mathbb{R}^{3}$. Indeed, let $\vartheta: \Omega \times \mathbb{R}^{+} \rightarrow \mathbb{R}$ be the absolute temperature of the conductor, $\vartheta_{0} \in \mathbb{R}^{+}$ the uniform equilibrium temperature and

$$
u(x, t)=\frac{\vartheta(x, t)-\vartheta_{0}}{\vartheta_{0}}
$$

the temperature variation field relative to the equilibrium reference value. According to the well-established theory due to Gurtin and Pipkin [9], we consider only small variations of the absolute temperature and the temperature gradient from equilibrium, namely,

$$
|u| \ll 1 \quad \text { and } \quad \frac{1}{\vartheta_{0}}|\nabla \vartheta|=|\nabla u| \ll 1
$$

and we suppose that the internal energy $e: \Omega \times \mathbb{R} \rightarrow \mathbb{R}$ and the heat flux vector $\mathbf{q}: \Omega \times \mathbb{R} \rightarrow \mathbb{R}^{3}$ are described by the following linear constitutive equations:

$$
\begin{aligned}
e(x, t) & =e_{0}+c_{0} u(x, t) \\
\mathbf{q}(x, t) & =-\int_{-\infty}^{t} k(t-s) \nabla u(x, s) d s
\end{aligned}
$$

where the heat flux memory kernel $k: \mathbb{R}^{+} \rightarrow \mathbb{R}$ is a sufficiently smooth, positive, summable function decreasing to zero at infinity. The positive constants $e_{0}$ and $c_{0}$ denote the internal energy at equilibrium and the specific heat, respectively. As usual, temperature evolution in a rigid heat conductor is governed by the energy balance equation. Here, assuming that the heat supply consists of a nonlinear temperature-dependent term, $-g(u)$ (accounting for certain types of laser induced radiative phenomena [10]), and a time-varying source $f$, the energy equation takes the form

$$
e_{t}(x, t)+\nabla \cdot \mathbf{q}(x, t)=f(x, t)-g(u(x, t)) .
$$

Taking $c_{0}=1$ and assuming the isothermal condition $\vartheta=\vartheta_{0}$ at the boundary $\partial \Omega$, we obtain (1.1), where $u_{0}$ represents the prescribed initial past history of $u$, which is assumed to vanish on $\partial \Omega$, as well as $u$.

Finally, we mention that (1.1) is suitable to describe other physical phenomena: for instance, the motion of a viscoelastic fluid in a tube, and the Olmstead model for reaction-diffusion processes in media with memory (see [13]).

At first glance, the hyperbolic nature of problem (1.1) in not apparent. Indeed, calling

$$
\beta=\int_{0}^{\infty} k(\sigma) d \sigma>0
$$

we may rewrite our equation as

$$
u_{t}-\beta \Delta u+\int_{0}^{\infty} k(\sigma) \Delta[u(t)-u(t-\sigma)] d \sigma+g(u)=f .
$$


So, if we neglect the hereditary term, we obtain a semilinear parabolic equation whose longtime behavior has been studied by many authors (see, for instance, $[1,16])$.

On the other hand, by differentiation with respect to time, equation (1.1) can be transformed into the second order integro-differential equation

$$
u_{t t}-k(0) \Delta u-\int_{0}^{\infty} k^{\prime}(\sigma) \Delta u(t-\sigma) d \sigma+g^{\prime}(u) u_{t}=f_{t}
$$

which reduces to a hyperbolic equation with nonlinear damping when the memory term is neglected and $k(0)$ is assumed to be positive. As a particular case, when the heat flux memory kernel takes the form

$$
k(s)=k_{0} \exp \left[-\frac{s}{\sigma_{0}}\right]
$$

(1.3) yields the following (hyperbolic) differential equation

$$
\sigma_{0} u_{t t}+\left[1+\sigma_{0} g^{\prime}(u)\right] u_{t}-\sigma_{0} k_{0} \Delta u+g(u)=f+\sigma_{0} f_{t}
$$

The hyperbolicity of (1.1) when $k(0)>0$ is properly expressed by the fact that the energy of a perturbation, initially given in a compact subset of $\Omega$, propagates with a finite speed $c \geq \sqrt{k(0)}$ (cf. [3]).

In the sequel we shall require the nonlinear term $g$ in (1.1) to comply some dissipativeness condition. Nevertheless an antidissipative behavior for small values of its argument will be allowed. For instance, if $g$ is a cubic-like function of the form $g(u)=u^{3}-\gamma u$ and the product $\gamma \sigma_{0}$ is large enough, then the coefficient of $u_{t}$ in (1.4) is negative for $|u|$ small. When this is the case, it is not necessarily true that bounded solutions converge to equilibria, and the dynamical behavior of the system is expected to be more complicated.

Concerning the heat flux memory kernel, we assume that there exists $\alpha>0$ such that, defining the kernel

$$
\mu(s)=-k^{\prime}(s)-\alpha k(s)
$$

the following hold:

$$
\begin{array}{ll}
\text { (h1) } & \mu \in C^{1}\left(\mathbb{R}^{+}\right) \cap L^{1}\left(\mathbb{R}^{+}\right) \\
\text {(h2) } & \mu(s) \geq 0 \quad \forall s \in \mathbb{R}^{+} \\
\text {(h3) } & \mu^{\prime}(s) \leq 0 \quad \forall s \in \mathbb{R}^{+} \\
\text {(h4) } & \mu^{\prime}(s)+\delta \mu(s) \leq 0 \quad \forall s \in \mathbb{R}^{+} \quad \text { and some } \quad \delta>0 \\
\text { (h5) } & k(s) \leq M \mu(s) \quad \forall s \in \mathbb{R}^{+} \quad \text { and some } \quad M>0 .
\end{array}
$$

It is readily seen that a kernel of the form $k(s)=k_{0} \exp \left[-\alpha_{0} s\right]$ fulfills (h1) $-(\mathrm{h} 5)$, for every $\alpha<\alpha_{0}$. In this case $M=1 /\left(\alpha_{0}-\alpha\right)$ and $\delta=\alpha$. 
We remark that condition (h4), which is not actually needed in the existence and uniqueness results that follow, implies the exponential decay of $\mu(s)$. Nonetheless, it allows $\mu(s)$ to have a singularity at $s=0$, whose order is less than 1.

The aim of this paper is the analysis of the asymptotic behavior of the solution of (1.1) together with its past history. For this reason, along the line of [2], we introduce a new variable which embodies the past history of the equation, namely

$$
\eta^{t}(x, s)=u(x, t)-u(x, t-s), \quad s \in \mathbb{R}^{+} .
$$

As a consequence, (1.2) reads

$$
u_{t}-\beta \Delta u+\int_{0}^{\infty} k(\sigma) \Delta \eta(\sigma) d \sigma+g(u)=f
$$

In order to focus on the dissipative and antidissipative terms of the problem, we need to handle a second order version of (1.6). This is achieved by substituting (1.5) into (1.3). Indeed, recalling that $k(0)=-\int_{0}^{\infty} k^{\prime}(\sigma) d \sigma$, we have

$$
u_{t t}+\int_{0}^{\infty} k^{\prime}(\sigma) \Delta \eta(\sigma) d \sigma+g^{\prime}(u) u_{t}=f_{t} .
$$

Then, addition of (1.7) and $\alpha$-times (1.6) leads to the system

$$
\left\{\begin{aligned}
u_{t t}= & \alpha \beta \Delta u-\alpha u_{t} \\
& +\int_{0}^{\infty} \mu(\sigma) \Delta \eta(\sigma) d \sigma-\alpha g(u)-g^{\prime}(u) u_{t}+\alpha f+f_{t} \\
\eta_{t}=- & \eta_{s}+u_{t} .
\end{aligned}\right.
$$

The second equation, needed to close the above system, is obtained differentiating (1.5).

Boundary and initial conditions are then translated into

$$
\left\{\begin{array}{l}
u(x, t)=0 \quad x \in \partial \Omega, \quad t \geq 0 \\
\eta^{t}(x, s)=0 \quad(x, s) \in \partial \Omega \times \mathbb{R}^{+}, \quad t \geq 0
\end{array}\right.
$$

and

where we set

$$
\left\{\begin{array}{l}
u(x, 0)=u_{0}(x) \quad x \in \Omega \\
u_{t}(x, 0)=v_{0}(x) \quad x \in \Omega \\
\eta^{0}(x, s)=\eta_{0}(x, s) \quad(x, s) \in \Omega \times \mathbb{R}^{+}
\end{array}\right.
$$

$$
\left\{\begin{array}{l}
u_{0}(x)=u_{0}(x, 0) \\
v_{0}(x)=\partial_{t} u_{0}(x, t)_{\mid t=0} \\
\eta_{0}(x, s)=u_{0}(x, 0)-u_{0}(x,-s) .
\end{array}\right.
$$

Existence, uniqueness and asymptotic behavior for the linear problem associated to (1.1), subject to initial-boundary conditions, have been investigated by several authors (e.g., $[7,8,11,12]$ ). In particular, in [7], we proved the 
exponential stability of the system along with the past summed history via semigroup techniques. The parabolic analogue to (1.1), obtained when $k$ has a Dirac delta distribution at the origin, has been considered in $[5,6]$, where we proved also the existence of a uniform attractor for the solutions.

The plan of the paper is as follows. In Section 2 we describe the functional setting. Section 3 is devoted to existence and uniqueness results. Finally, in Section 4, we prove the existence of a uniform absorbing set for the solutions.

\section{The functional setting}

Let $\Omega \subset \mathbb{R}^{3}$ be a bounded domain with smooth boundary. With usual notation, we introduce the spaces $L^{p}, W^{k, p}, H^{k}=W^{k, 2}$ and $H_{0}^{k}$ acting on $\Omega$. Throughout the paper, we denote by $c$ a generic positive constant (which may vary even in the same line). Given a space $\mathcal{X}$, we denote its norm by $\|\cdot\|_{\mathcal{X}}$ and its inner product by $\langle\cdot, \cdot\rangle_{\mathcal{X}}$ (summation on the components is understood when we have vectorial quantities). When $\mathcal{X}=L^{2}$ we omit the subscript. The symbol $\langle\cdot, \cdot\rangle$ will be also used to denote the duality map between $H^{-1}$ and $H_{0}^{1}$ or between $L^{p}$ and $L^{q}$. We will also consider spaces of $\mathcal{X}$-valued functions defined on an (possibly infinite) interval $I$ such as $C(I, \mathcal{X}), L^{p}(I, \mathcal{X})$ and $W^{k, p}(I, \mathcal{X})$, with the usual norms. In force of Poincaré inequality

$$
\|u\|^{2} \leq \lambda_{0}\|\nabla u\|^{2} \quad \forall u \in H_{0}^{1}
$$

(for some $\lambda_{0}>0$ ) the inner product in $H_{0}^{1}$ will be chosen to be

$$
\langle\cdot, \cdot\rangle_{H_{0}^{1}}=\langle\nabla \cdot, \nabla \cdot\rangle .
$$

In view of $(\mathrm{h} 1)-(\mathrm{h} 2)$, let $\mathcal{M}=L_{\mu}^{2}\left(\mathbb{R}^{+}, H_{0}^{1}\right)$ be the Hilbert space of $H_{0}^{1}$-valued functions on $\mathbb{R}^{+}$, endowed with the inner product

$$
\langle\varphi, \psi\rangle_{\mathcal{M}}=\int_{0}^{\infty} \mu(\sigma)\langle\nabla \varphi(s), \nabla \psi(\sigma)\rangle d \sigma
$$

Finally we introduce the Hilbert space

$$
\mathcal{H}=H_{0}^{1} \times L^{2} \times \mathcal{M} .
$$

To describe the asymptotic behavior of the solutions of our system we need also to introduce the Banach space $\mathcal{T}$ of $W_{\text {loc }}^{1,1}$ translation bounded $L^{2}$-valued functions on $\mathbb{R}^{+}$, namely

$$
\mathcal{T}=\left\{f \in W_{\mathrm{loc}}^{1,1}\left(\mathbb{R}^{+}, L^{2}\right):\|f\|_{\mathcal{T}}=\sup _{\xi \geq 0} \int_{\xi}^{\xi+1}\|f(y)\|+\left\|f^{\prime}(y)\right\| d y<\infty\right\} .
$$

We conclude the section with a slight generalization of Lemma A.3 in [14]. 
Lemma 2.1 Let $h \in C(\mathbb{R})$ satisfy $|h(u)| \leq c\left(1+|u|^{p}\right)$, for some $c>0$ and some $p \geq 0$, and let $X$ be a bounded Lebesgue measurable subset of $\mathbb{R}^{n}$. Then for every $q \geq \max \{1,2 p\}$, and every $r \geq 0 h$ is a continuous mapping from $L^{q+2 r}(X)$ to $L^{2+r}(X)$.

\section{$3 \quad$ Existence and uniqueness}

We assume that the nonlinear term $g$ is a continuously differentiable function on $\mathbb{R}$. Moreover, there exist $c_{1}, c_{2}>0$ and $0 \leq p<2$ such that

$$
\begin{array}{ll}
\text { (g1) } & g^{\prime}(y) \geq-c_{1} \\
\text { (g2) } & \left|g^{\prime}(y)\right| \leq c_{2}\left(1+|y|^{p}\right) .
\end{array}
$$

Remark 3.1 Let us rewrite the first equation in (1.8) as follows:

$$
u_{t t}+\left(\alpha+g^{\prime}(u)\right) u_{t}-\alpha \beta \Delta u=\int_{0}^{\infty} \mu(\sigma) \Delta \eta(\sigma) d \sigma-\alpha g(u)+\alpha f+f_{t} .
$$

If the constant $c_{1}$ in (g1) satisfies

$$
c_{1}<\alpha
$$

then it is apparent that

$$
\inf _{y \in \mathbb{R}}\left(\alpha+g^{\prime}(y)\right)>0
$$

and the damping term in the left-hand side of (3.1) furnishes a significant (and non-degenerate) contribution to energy dissipation. Notice that condition (3.2) is not used to prove existence and uniqueness results. Nevertheless, it plays a crucial role in the proof of the existence of uniform absorbing sets.

Definition 3.2 Let (h1)-(h2) hold. Set $I=[0, T]$, for $T>0$, and let $f \in$ $W^{1,1}\left(I, L^{2}\right)$. We say a function $\left(u, u_{t}, \eta\right) \in C(I, \mathcal{H})$ is a solution to problem $(1.8)-$ (1.9) in the time interval $I$, with initial data $\left(u_{0}, v_{0}, \eta_{0}\right) \in \mathcal{H}$, provided

$$
\begin{aligned}
\left\langle u_{t t}, w\right\rangle= & -\alpha \beta\langle\nabla u, \nabla w\rangle-\alpha\left\langle u_{t}, w\right\rangle-\int_{0}^{\infty} \mu(\sigma)\langle\nabla \eta(\sigma), \nabla w\rangle d \sigma \\
& -\alpha\langle g(u), w\rangle-\left\langle g^{\prime}(u) u_{t}, w\right\rangle+\alpha\langle f, w\rangle+\left\langle f_{t}, w\right\rangle \\
\left\langle\eta_{t}+\eta_{s}, \varphi\right\rangle_{\mathcal{N}}= & \left\langle u_{t}, \varphi\right\rangle_{\mathcal{N}}
\end{aligned}
$$

for all $w \in H_{0}^{1}, \varphi \in \mathcal{N}$, and a.e. $t \in I$, where we set $\mathcal{N}=L_{\mu}^{2}\left(\mathbb{R}^{+}, L^{2}\right)$. Here, $-\eta_{s}$ is interpreted as the infinitesimal generator of the right-translation semigroup on $\mathcal{M}$.

We now state and prove existence and uniqueness results.

Theorem 3.3 (Existence). Let (h1)-(h3) and $(\mathrm{g} 1)-(\mathrm{g} 2)$ hold. Then, given any $T>0$, problem (1.8)-(1.9) has a solution $\left(u, u_{t}, \eta\right)$ in the time interval $I=[0, T]$, with initial data $\left(u_{0}, v_{0}, \eta_{0}\right)$. 
Proof. The theorem is proved re-casting exactly the Faedo-Galerkin scheme used in [5]. Uniform estimates for the approximate solutions are obtained as in the proof of the following Theorem 4.2. Actually the situation here is much simpler, since the nonlinear terms are controlled observing that, for every $u \in H_{0}^{1}$ and $v \in L^{2}$

$$
\left\langle g^{\prime}(u) v, v\right\rangle \geq-c_{1}\|v\|^{2}
$$

and

$$
|\langle g(u), v\rangle| \leq\|g(u)\|\|v\| \leq c\left(1+\|\nabla u\|^{2}+\|v\|^{2}\right)
$$

in force of (g1)-(g2) and Young inequality. Thus, for a sequence of approximate solutions $\left(u_{n}, \eta_{n}\right)$, one gets the uniform bound

$$
\left\|u_{n}\right\|_{L^{\infty}\left(I, H_{0}^{1}\right) \cap W^{1, \infty}\left(I, L^{2}\right)} \leq c .
$$

Notice that, since $f \in W^{1,1}\left(I, L^{2}\right)$, a generalized Gronwall lemma in the differential form is required (see, e.g., Lemma A.1 in [14]). Concerning passage to limit, the only problem is the nonlinear term $\alpha g(u)-g^{\prime}(u) u_{t}$. Exploiting classical compact embeddings (recall that $p<2$ ) we conclude that, up to a subsequence,

$$
u_{n} \longrightarrow u \quad \text { strongly in } L^{2(p+1)}(I \times \Omega) \text {. }
$$

Moreover,

$$
\partial_{t} u_{n} \longrightarrow u_{t} \quad \text { weakly in } L^{2}(I \times \Omega) .
$$

Therefore, in virtue of (g2) and (3.4), applying Lemma 2.1 we get the convergences

$$
g\left(u_{n}\right) \longrightarrow g(u) \quad \text { strongly in } L^{2}(I \times \Omega)
$$

and

$$
g^{\prime}\left(u_{n}\right) \longrightarrow g^{\prime}(u) \quad \text { strongly in } L^{3}(I \times \Omega) .
$$

Let now $w \in H_{0}^{1}$. From (3.6) it is apparent that

$$
\left\langle g\left(u_{n}\right), w\right\rangle \longrightarrow\left\langle g\left(u_{n}\right), w\right\rangle .
$$

Convergence (3.7) entails

$$
g^{\prime}\left(u_{n}\right) w \longrightarrow g^{\prime}\left(u_{n}\right) w \quad \text { strongly in } L^{2}(I \times \Omega)
$$

which, together with (3.5), gives

$$
\left\langle g^{\prime}\left(u_{n}\right) \partial_{t} u_{n}, w\right\rangle \longrightarrow\left\langle g^{\prime}(u) u_{t}, w\right\rangle .
$$

Continuity in time of $u$ and $u_{t}$ follows from usual arguments (cf. [4]). Continuity of $\eta$ follows consequently, as in the proof of Theorem 3.2 in [15]. 
Theorem 3.4 (Uniqueness). Let (h1)-(h3) and (g1)-(g2) hold. Then, given any $T>0$, the solution $\left(u, u_{t}, \eta\right)$ to (1.8)-(1.9) in the time interval $I=[0, T]$, with initial data $\left(u_{0}, v_{0}, \eta_{0}\right)$ is unique.

Proof. For $i=1,2$, let $z_{i}=\left(u_{i}, \partial_{t} u_{i}, \eta_{i}\right)$ be two solutions of (1.8)-(1.9) with initial data $z_{0}=\left(u_{0}, v_{0}, \eta_{0}\right)$, and denote $z=\left(u, u_{t}, \eta\right)=z_{1}-z_{2}$, with $z(0)=(0,0,0)$. Adding and subtracting in (1.8) we obtain

$$
\begin{aligned}
u_{t t}= & \alpha \beta \Delta u-\alpha u_{t}+\int_{0}^{\infty} \mu(\sigma) \Delta \eta(\sigma) d \sigma-\alpha\left[g\left(u_{1}\right)-g\left(u_{2}\right)\right] \\
& -\partial_{t}\left[g\left(u_{1}\right)-g\left(u_{2}\right)\right] \\
\eta_{t}= & -\eta_{s}+u_{t} .
\end{aligned}
$$

Fix then $\tau \in(0, T]$, and define

$$
v(t)= \begin{cases}\int_{t}^{\tau} u(y) d y & 0 \leq t \leq \tau \\ 0 & \tau \leq t \leq T .\end{cases}
$$

Moreover, let

$$
\tilde{u}(t)=\int_{0}^{t} u(y) d y \quad \text { and } \quad \tilde{\eta}^{t}=\int_{0}^{t} \eta^{y} d y .
$$

Notice that, for every $t \in I, v(t) \in H_{0}^{1}, \tilde{u}(t) \in H_{0}^{1}, \tilde{\eta}^{t} \in \mathcal{M}$, and $v(\tau)=\tilde{u}(0)=$ $\tilde{\eta}^{0}=0$. Finally, $v_{t}=-u, \tilde{u}_{t}=u$, and $\tilde{\eta}_{t}=\eta$. Take the duality product of $(3.8)$ with $v(t)$, and integrate in time from 0 to $\tau$. Thanks to the above conditions, repeated integrations by parts lead to

$$
\begin{aligned}
\frac{1}{2}\left(\|u(\tau)\|^{2}+\alpha \beta\|\nabla \tilde{u}(\tau)\|^{2}\right)+\alpha \int_{0}^{\tau}\|u(t)\|^{2} d t \\
=-\int_{0}^{\tau}\left\langle\tilde{\eta}^{t}, u(t)\right\rangle_{\mathcal{M}} d t-\alpha \int_{0}^{\tau}\left\langle g\left(u_{1}(t)\right)-g\left(u_{2}(t)\right), v(t)\right\rangle d t \\
\quad-\int_{0}^{\tau}\left\langle g\left(u_{1}(t)\right)-g\left(u_{2}(t)\right), u(t)\right\rangle d t .
\end{aligned}
$$

In force of (g2), and the fact that $u_{i} \in L^{\infty}\left(I, H_{0}^{1}\right)$, we get

$$
\left\|g\left(u_{1}\right)-g\left(u_{2}\right)\right\|_{L^{6 / 5}} \leq\left\|c_{2}\left(1+\left|u_{1}\right|^{p}+\left|u_{2}\right|^{p}\right)\right\|_{L^{3}}\|u\| \leq c\|u\| .
$$

Also, observe that

$$
\begin{aligned}
\int_{0}^{\tau}\|\nabla v(t)\|^{2} d t & =\int_{0}^{\tau}\|\nabla \tilde{u}(\tau)-\nabla \tilde{u}(t)\|^{2} d t \\
& \leq 2 \tau\|\nabla \tilde{u}(\tau)\|^{2}+2 \int_{0}^{\tau}\|\nabla \tilde{u}(t)\|^{2} d t
\end{aligned}
$$


Therefore, the continuous embedding $L^{6 / 5} \hookrightarrow H^{-1}$, Hölder inequality, and Young inequality, entail

$$
\begin{aligned}
& -\alpha \int_{0}^{\tau}\left\langle g\left(u_{1}(t)\right)-g\left(u_{2}(t)\right), v(t)\right\rangle d t \\
& \quad \leq \alpha \int_{0}^{\tau}\left\|g\left(u_{1}(t)\right)-g\left(u_{2}(t)\right)\right\|_{H^{-1}}\|\nabla v(t)\| d t \\
& \leq c \int_{0}^{\tau}\|u(t)\|\|\nabla v(t)\| d t \\
& \quad \leq \frac{\alpha \beta}{4}\|\nabla \tilde{u}(\tau)\|^{2}+c \int_{0}^{\tau}\|\nabla \tilde{u}(t)\|^{2} d t+c \int_{0}^{\tau}\|u(t)\|^{2} d t .
\end{aligned}
$$

Concerning the last term of (3.10), condition (g1) yields

$$
-\int_{0}^{\tau}\left\langle g\left(u_{1}(t)\right)-g\left(u_{2}(t)\right), u(t)\right\rangle d t \leq c_{1} \int_{0}^{\tau}\|u(t)\|^{2} d t .
$$

We now integrate equality (3.9) from 0 to $t$, to get

$$
\eta^{t}+\tilde{\eta}_{s}^{t}=u(t)
$$

Taking the inner product in $\mathcal{M}$ of the above equation and $\tilde{\eta}^{t}$, and integrating in time from 0 to $\tau$, we have

$$
\int_{0}^{\tau}\left\langle\eta^{t}+\tilde{\eta}_{s}^{t}, \tilde{\eta}^{t}\right\rangle_{\mathcal{M}} d t=\int_{0}^{\tau}\left\langle u(t), \tilde{\eta}^{t}\right\rangle_{\mathcal{M}} d t .
$$

Exploiting (h3), integration by parts, and an approximation argument (cf. $[5,15])$ the integrand of the left-hand side of (3.13) is seen to satisfy

$$
\left\langle\eta^{t}+\tilde{\eta}_{s}^{t}, \tilde{\eta}^{t}\right\rangle_{\mathcal{M}}=\left\langle\tilde{\eta}_{t}^{t}+\tilde{\eta}_{s}^{t}, \tilde{\eta}^{t}\right\rangle_{\mathcal{M}} \geq \frac{1}{2} \frac{d}{d t}\left\|\tilde{\eta}^{t}\right\|_{\mathcal{M}}^{2}
$$

and (3.13) turns into

$$
\frac{1}{2}\left\|\tilde{\eta}^{\tau}\right\|_{\mathcal{M}}^{2} \leq \int_{0}^{\tau}\left\langle u(t), \tilde{\eta}^{t}\right\rangle_{\mathcal{M}} d t .
$$

Finally, addition of (3.10) and (3.14), with the aid of (3.11)-(3.13), entails

$$
\|u(\tau)\|^{2}+\|\nabla \tilde{u}(\tau)\|^{2}+\left\|\tilde{\eta}^{\tau}\right\|_{\mathcal{M}}^{2} \leq c \int_{0}^{\tau}\|u(t)\|^{2} d t+c \int_{0}^{\tau}\|\nabla \tilde{u}(t)\|^{2} d t
$$

and Gronwall lemma in the integral form implies that $u(\tau)=\tilde{\eta}^{\tau}=0$. Since $\tau$ is arbitrary, we conclude that $\left(u(t), v(t), \eta^{t}\right)=(0,0,0)$ for every $t \in I$. 
Remark 3.5 For the solution $z=\left(u, u_{t}, \eta\right)$ with initial data $z_{0}$ of (1.8)-(1.9) define

$$
\mathcal{U} z(t)=u_{t}(t)-\beta \Delta u(t)+\int_{0}^{\infty} k(\sigma) \Delta \eta^{t}(\sigma) d \sigma+g(u(t))-f(t)
$$

When (h5) holds too, by Lemma 2.1 we get the continuity $\mathcal{U} z \in C\left(I, H^{-1}\right)$. In particular, $\mathcal{U} z(0)=\mathcal{U} z_{0}$. Since by definition

$$
\partial_{t} \mathcal{U} z+\alpha \mathcal{U} z=0
$$

we conclude that

$$
\mathcal{U} z(t)=\mathcal{U} z_{0} e^{-\alpha t}
$$

Thus $z$ solves (1.6) provided that $\mathcal{U} z_{0}=0$. This condition is not really a constraint on the initial data; indeed equation (1.6) is of the first order in time, and the initial value of $u_{t}$ is automatically determined by the equation. Conversely, every $z \in C(I, \mathcal{H})$ which solves (1.6) is a solution of (1.8)-(1.9). Hence, given $u_{0} \in H_{0}^{1}$ and $\eta_{0} \in \mathcal{M}$ there is a unique solution $z \in C(I, \mathcal{H})$ of $(1.6)$ if and only if the vector $v_{0}$ determined by the equation $\mathcal{U}\left(u_{0}, v_{0}, \eta_{0}\right)=0$ belongs to $L^{2}$.

In the sequel, we agree to denote the solution $z(t)$ of (1.8)-(1.9) with initial data $z_{0}$ by $S(t) z_{0}$. In force of the existence and continuous dependence results, the one-parameter family of operators $S(t)$ enjoys the following properties:

(i) $S(0)$ is the identity map on $\mathcal{H}$

(ii) $S(t) z \in C([0, \infty), \mathcal{H})$ for any $z \in \mathcal{H}$.

When the system is autonomous ( $f$ independent of time) $S(t)$ fulfills also

(iii) $S(t) S(\tau)=S(t+\tau)$ for any $t, \tau \geq 0$.

We remark that $S(t)$ might not be a $C_{0}$-semigroup of continuous (nonlinear) operators on $\mathcal{H}$, since the continuity $S(t) \in C(\mathcal{H}, \mathcal{H})$ for any $t \geq 0$, in general, does not hold, unless we are in the simpler situation when $g$ is Lipschitz.

Theorem 3.6 Let (h1)-(h3) and (g1)-(g2) with $p=0$ hold (that is, $g$ is Lipschitz). Then $S(t) \in C(\mathcal{H}, \mathcal{H})$ for any $t \geq 0$. In particular, if $f$ is independent of time, $S(t)$ is a $C_{0}$-semigroup.

Proof. Let $z_{0 n} \in \mathcal{H}$ be a sequence converging in $\mathcal{H}$ to $z_{0 \infty} \in \mathcal{H}$. Denote by $z_{n}=\left(u_{n}, \partial_{t} u_{n}, \eta_{n}\right)$ and $z_{\infty}=\left(u_{\infty}, \partial_{t} u_{\infty}, \eta_{\infty}\right)$ the corresponding solutions to (1.8)-(1.9). Finally, let $\bar{z}_{0 n}=z_{0 n}-z_{0 \infty}$ and $\bar{z}_{n}=z_{n}-z_{\infty}=\left(\bar{u}_{n}, \partial_{t} \bar{u}_{n}, \bar{\eta}_{n}\right)$. Again, we add and subtract in (1.8), and we multiply the two resulting equation by $\partial_{t} \bar{u}_{n}$ in $L^{2}$, and by $\bar{\eta}_{n}$ in $\mathcal{M}$, respectively. Clearly, the multiplications make sense for Faedo-Galerkin approximants. However, due to the uniqueness result, the final estimates hold to the limit. Adding the results, and exploiting the inequality

$$
\left\langle g^{\prime}\left(u_{n}\right) \partial_{t} u_{n}-g^{\prime}\left(u_{\infty}\right) \partial_{t} u_{\infty}, \partial_{t} \bar{u}_{n}\right\rangle \leq c\left\|\partial_{t} u_{n}\right\|^{2}+c\left\|\left(g^{\prime}\left(u_{n}\right)-g^{\prime}\left(u_{\infty}\right)\right) \partial_{t} u_{\infty}\right\|^{2}
$$


we easily obtain

$$
\frac{d}{d t}\left\|\bar{z}_{n}\right\|_{\mathcal{H}}^{2} \leq c\left\|\bar{z}_{n}\right\|_{\mathcal{H}}^{2}+c\left\|\left(g^{\prime}\left(u_{n}\right)-g^{\prime}\left(u_{\infty}\right)\right) \partial_{t} u_{\infty}\right\|^{2}
$$

An immediate generalization of Theorem 3.4 (i.e., taking different initial data) shows that, as $z_{0 n} \rightarrow z_{0 \infty}$ in $\mathcal{H}$, the convergence $u_{n} \rightarrow u_{\infty}$ holds in $L^{2}(I \times \Omega)$. Hence, there exists a subsequence $u_{n_{k}} \rightarrow u_{\infty}$ a.e. in $I \times \Omega$. Setting $\psi_{k}=\left(g^{\prime}\left(u_{n_{k}}\right)-\right.$ $\left.g^{\prime}\left(u_{\infty}\right)\right) \partial_{t} u_{\infty}$, we have that

$$
\varepsilon_{k}=\int_{0}^{T} \int_{\Omega}\left|\psi_{k}\right|^{2} d x d t \longrightarrow 0 \quad(k \rightarrow \infty)
$$

by virtue of the Lebesgue dominated convergence theorem. Thus Gronwall lemma applied to the subsequence $\bar{z}_{n_{k}}$ yields

$$
\left\|\bar{z}_{n_{k}}(t)\right\|_{\mathcal{H}}^{2} \leq e^{c T}\left\|\bar{z}_{0 n_{k}}\right\|_{\mathcal{H}}^{2}+c e^{c T} \varepsilon_{k}
$$

for any $t \in I$. We conclude that, whenever $z_{0 n} \rightarrow z_{0 \infty}$ in $\mathcal{H}$, there exists a subsequence $z_{0 n_{k}}$ such that $S(t) z_{0 n_{k}} \rightarrow S(t) z_{0 \infty}$ for all $t \in I$. Using an immediate contradiction argument, this implies that $S(t) z_{0 n} \rightarrow S(t) z_{0 \infty}$ for any $t \in I$. Being $T$ arbitrary, we proved that $S(t) \in C(\mathcal{H}, \mathcal{H})$ for any $t \geq 0$.

\section{Existence of uniform absorbing sets}

An absorbing set for $S(t)$ is a bounded set $\mathcal{B}_{0} \subset \mathcal{H}$ such that for any bounded set $\mathcal{B} \subset \mathcal{H}$ there exists a time $t^{*}=t^{*}(\mathcal{B})$ such that

$$
S(t) \mathcal{B} \subset \mathcal{B}_{0} \quad \forall t \geq t^{*} .
$$

To stress the dependence of $S(t)$ on the given external term $f$, we shall write $S_{f}(t)$. The aim of this section is to prove the existence of an absorbing set for $S_{f}(t)$, which is uniform as $f$ is allowed to run in a certain functional set. In order to accomplish that, we are required to ask stronger conditions both on the nonlinear term and on the memory kernel.

Concerning the nonlinear term, setting

$$
G(y)=\int_{0}^{y} g(\xi) d \xi
$$

we assume that the following hold (cf. [4]):

$$
\begin{array}{ll}
\text { (g3) } & \liminf _{|y| \rightarrow \infty} \frac{G(y)}{y^{2}} \geq 0 \\
\text { (g4) } & \liminf _{|y| \rightarrow \infty} \frac{y g(y)-c_{3} G(y)}{y^{2}} \geq 0
\end{array}
$$


for some $c_{3}>0$. There is no loss of generality if we assume $c_{3} \leq \alpha \beta / 2$. These conditions are fulfilled by many classical examples, such as $g(u)=|u|^{\rho} u-\gamma u$ or $g(u)=\sin u($ see $[16])$. For $u \in H_{0}^{1}$, denote

$$
\mathcal{G}(u)=\int_{\Omega} G(u(x)) d x .
$$

The easy proof of next lemma is left to the reader.

Lemma 4.1 Assume (g3)-(g4). Then for every $\nu>0$ there exist $c(\nu)>0$ such that

$$
\begin{aligned}
\mathcal{G}(u) & \geq-\nu\|\nabla u\|^{2}-c(\nu) \\
\langle g(u), u\rangle & \geq-\nu\|\nabla u\|^{2}-c(\nu) \\
\langle g(u), u\rangle-c_{3} \mathcal{G}(u) & \geq-\nu\|\nabla u\|^{2}-c(\nu)
\end{aligned}
$$

for all $u \in H_{0}^{1}$.

Theorem 4.2 Assume (h1)-(h4), (g1)-(g4), and (3.2) (cf. Remark 3.1). Let $\mathcal{F} \subset \mathcal{T}$ be a bounded set. Then there exists an absorbing set for $S_{f}(t)$ which is uniform as $f \in \mathcal{F}$.

Proof. For every $r>0$, let $\mathcal{B}(r)$ be the ball of $\mathcal{H}$ of radius $r$ centered in the origin. Moreover, denote

$$
M_{0}=\sup _{h \in \mathcal{F}}\|h\|_{\mathcal{T}} .
$$

Fix then $R>0$, and let $f \in \mathcal{F}$ and $z_{0} \in \mathcal{B}(R)$. For any $0<\varepsilon \leq \alpha / 2$, introduce the new variable $w=u_{t}+\varepsilon u$; then multiply the first equation of (1.8) by $w$ in $\mathcal{H}$, and the second one by $\eta$ in $\mathcal{M}$, where $\left(u(t), u_{t}(t), \eta^{t}\right)=S_{f}(t) z_{0}$. Clearly, the multiplication makes sense in a Faedo-Galerkin scheme. So we get

$$
\begin{aligned}
& \frac{1}{2} \frac{d}{d t}\left(\alpha \beta\|\nabla u\|^{2}+\|w\|^{2}+2(\alpha-\varepsilon) \mathcal{G}(u)+2 \varepsilon\langle g(u), u\rangle\right) \\
& \quad+\varepsilon \alpha \beta\|\nabla u\|^{2}+(\alpha-\varepsilon)\|w\|^{2}=-\int_{0}^{\infty} \mu(\sigma)\left\langle\nabla \eta(\sigma), \nabla u_{t}\right\rangle d \sigma-\varepsilon \\
& \quad \int_{0}^{\infty} \mu(\sigma)\langle\nabla \eta(\sigma), \nabla u\rangle d \sigma-\varepsilon \alpha\langle g(u), u\rangle \\
& \quad-\left\langle g^{\prime}(u) u_{t}, u_{t}\right\rangle+\varepsilon(\alpha-\varepsilon)\langle u, w\rangle+\alpha\langle f, w\rangle+\left\langle f_{t}, w\right\rangle
\end{aligned}
$$

and

$$
\frac{1}{2} \frac{d}{d t}\|\eta\|_{\mathcal{M}}^{2}+\frac{\delta}{2}\|\eta\|_{\mathcal{M}}^{2} \leq \int_{0}^{\infty} \mu(\sigma)\left\langle\nabla \eta(\sigma), \nabla u_{t}\right\rangle d \sigma .
$$

The above inequality (4.5) is obtained integrating by parts the term

$$
\int_{0}^{\infty} \mu(\sigma)\left\langle\nabla \eta_{s}(\sigma), \nabla \eta(\sigma)\right\rangle d \sigma=\frac{1}{2} \int_{0}^{\infty} \mu(\sigma) \frac{d}{d s}\|\nabla \eta(\sigma)\|^{2} d \sigma
$$


and using (h4) (see [5] for more details). Setting $c_{4}=\|\mu\|_{L^{1}\left(\mathbb{R}^{+}\right)} / \delta$, Young inequality entails

$$
-\varepsilon \int_{0}^{\infty} \mu(\sigma)\langle\nabla \eta(\sigma), \nabla u\rangle d \sigma \leq \varepsilon^{2} c_{4}\|\nabla u\|^{2}+\frac{\delta}{4}\|\eta\|_{\mathcal{M}}^{2}
$$

Denote

$$
\tau=\tau(\varepsilon)=\frac{\alpha}{\alpha-\varepsilon+\varepsilon c_{3}} .
$$

Notice that $0<\tau \leq 2$. In particular we see that $\alpha \beta \geq \tau c_{3}$. Making use of (4.3), with $\nu=\beta / 4$,

$$
\begin{aligned}
-\varepsilon \alpha\langle g(u), u\rangle & =-\varepsilon \tau(\alpha-\varepsilon)\langle g(u), u\rangle-\varepsilon^{2} \tau c_{3}\langle g(u), u\rangle \\
& \leq-\varepsilon \tau c_{3}(\alpha-\varepsilon) \mathcal{G}(u)-\varepsilon^{2} \tau c_{3}\langle g(u), u\rangle+\frac{\varepsilon \alpha \beta}{4}\|\nabla u\|^{2}+c_{5}
\end{aligned}
$$

with $c_{5}=\alpha^{2} c(\beta / 4)$. Condition (g1), (2.1), and Young inequality, entail

$$
\begin{aligned}
& -\left\langle g^{\prime}(u) u_{t}, u_{t}\right\rangle+\varepsilon(\alpha-\varepsilon)\langle u, w\rangle \\
& \quad \leq c_{1}\left\|u_{t}\right\|^{2}+\varepsilon \alpha\|u\|\|w\| \\
& \quad \leq c_{1}\|w\|^{2}+\varepsilon^{2} c_{1}\|u\|^{2}+\varepsilon\left(2 c_{1}+\alpha\right)\|u\|\|w\| \\
& \quad \leq\left(c_{1}+\frac{\sqrt{\varepsilon}\left(2 c_{1}+\alpha\right)}{2}\right)\|w\|^{2}+\varepsilon \sqrt{\varepsilon} \lambda_{0}\left(\frac{2 c_{1}+\alpha+\alpha \sqrt{2 \alpha}}{2}\right)\|\nabla u\|^{2} .
\end{aligned}
$$

Finally,

$$
\alpha\langle f, w\rangle+\left\langle f_{t}, w\right\rangle \leq(\alpha+1)\left(\|f\|+\left\|f_{t}\right\|\right)\|w\| .
$$

Due to (4.1)-(4.2) it is apparent that there exist $c_{6}>0$ such that, defining

$$
\Phi^{2}=\alpha \beta\|\nabla u\|^{2}+\|w\|^{2}+\|\eta\|_{\mathcal{M}}^{2}+2(\alpha-\varepsilon) \mathcal{G}(u)+2 \varepsilon\langle g(u), u\rangle+c_{6}
$$

the relation

$$
\Phi^{2} \geq c_{7}\left\|S_{f}(t) z_{0}\right\|^{2}
$$

holds for some $c_{7}>0$ and every $\varepsilon$ small enough. Moreover, from (g2), there exists $\Lambda(r)>0$ such that

$$
\Phi^{2}(0) \leq \Lambda(r) \quad \text { whenever } \quad\left\|z_{0}\right\|_{\mathcal{H}}^{2} \leq r .
$$

Choose now $\varepsilon \leq \alpha / 2$ small enough such that (4.10) and the following inequalities hold:

$$
\begin{gathered}
\frac{\delta}{2} \geq \varepsilon \tau c_{3} \\
\frac{3 \alpha \beta}{4}-\varepsilon c_{4}-\sqrt{\varepsilon} \lambda_{0}\left(\frac{2 c_{1}+\alpha+\alpha \sqrt{2 \alpha}}{2}\right) \geq \frac{\tau c_{3}}{2}
\end{gathered}
$$


and

$$
\alpha-c_{1}-\varepsilon-\frac{\sqrt{\varepsilon}\left(2 c_{1}+\alpha\right)}{2} \geq \frac{\varepsilon \tau c_{3}}{2} .
$$

Hence, setting

$$
\varepsilon_{0}=\varepsilon \tau c_{3} \quad \text { and } \quad c_{8}=2 c_{5}+\varepsilon_{0} c_{6}
$$

adding (4.4)-(4.5), and collecting (4.6)-(4.9) and (4.12)-(4.14), we are led to

$$
\frac{d}{d t} \Phi^{2}+\varepsilon_{0} \Phi^{2} \leq c_{8}+(\alpha+1)\left(\|f\|+\left\|f_{t}\right\|\right) \Phi .
$$

Applying a generalization of Gronwall lemma to (4.15) (see, for instance, $[14,15]$ ), we get the inequality

$$
\Phi^{2}(t) \leq 2 \Phi^{2}(0) e^{-\varepsilon_{0} t}+C\left(M_{0}\right) \quad \forall t \in \mathbb{R}^{+}
$$

where

$$
C\left(M_{0}\right)=\frac{2 c_{8}}{\varepsilon_{0}}+\frac{e^{\varepsilon_{0}} M_{0}^{2}(\alpha+1)^{2}}{\left(1-e^{-\varepsilon_{0} / 2}\right)^{2}} .
$$

Therefore from (4.10)-(4.11) and (4.16),

$$
\left\|S_{f}(t) z_{0}\right\|_{\mathcal{H}}^{2} \leq \frac{2 \Lambda\left(R^{2}\right)}{c_{7}} e^{-\varepsilon_{0} t}+\frac{C\left(M_{0}\right)}{c_{7}} \quad \forall t \in \mathbb{R}^{+}
$$

and this relation holds for every $z_{0} \in \mathcal{B}(R)$ and $f \in \mathcal{F}$. Setting

$$
\mathcal{B}_{0}=\mathcal{B}\left(\sqrt{2 C\left(M_{0}\right) / c_{7}}\right)
$$

and

$$
t^{*}=t^{*}(R)=\max \left\{\frac{1}{\varepsilon_{0}} \log \left[\frac{C\left(M_{0}\right)}{2 \Lambda\left(R^{2}\right)}\right], 0\right\}
$$

we conclude that

$$
\bigcup_{f \in \mathcal{F}} S_{f}(t) \mathcal{B}(R) \subset \mathcal{B}_{0} \quad \forall t \geq t^{*}
$$

as desired.

\section{References}

[1] A.V. BABIN, M.I. VISHIK, Attractors of evolution equations. North-Holland, Amsterdam, 1992.

[2] C.M. DAfERMOS, Asymptotic stability in viscoelasticity, Arch. Rational Mech. Anal. 37 (1970), 297-308.

[3] M. FABRIZIO, G. GENTILI, D.W. REYNOLDS, On a rigid linear heat conductor with memory, Internat. J. Engrg. Sci. 36 (1998), 765-782. 
Vol. 8, 2001 Asymptotic behavior of a nonlinear hyperbolic heat equation with memory 171

[4] J.M. GHIDAGLIA, R. TEMAM, Attractors for damped nonlinear hyperbolic equations, J. Math. Pures Appl. 66 (1987), 273-319.

[5] C. GIORGI, A. MARZOCCHI, V. PATA, Asymptotic behavior of a semilinear problem in heat conduction with memory, NoDEA Nonlinear Differential Equations Appl. 5 (1998), 333-354.

[6] C. GIORGI, A. MARZOCCHI, V. PATA, Uniform attractors for a nonautonomous semilinear heat equation with memory, Quart. Appl. Math. 58 (2000), 661-683.

[7] C. GIORGI, M.G. NASO, V. PATA, Exponential stability in linear heat conduction with memory: a semigroup approach, Comm. Appl. Anal. 5 (2001), $121-134$.

[8] H. GRABMÜLLER, On linear theory of heat conduction in materials with memory, Proc. Roy. Soc. Edinburgh A-76 (1976-77), 119-137.

[9] M.E. GURTIN, A.C. PIPKIN, A general theory of heat conduction with finite wave speeds, Arch. Rational Mech. Anal. 31 (1968), 113-126.

[10] Y.I. LYSIKOV, On the possibility of development of vibrations during heating of the transparent dielectric by optical radiation, Zh. Prikl. Math. $i$ Tekh. Fiz. 4 (1984), 56-59.

[11] R.K. MILLER, An integrodifferential equation for rigid heat conductors with memory, J. Math. Anal. Appl. 66 (1978), 331-332.

[12] J.W. NUNZIATO, On heat conduction in materials with memory, Quart. Appl. Math. 29 (1971), 187-204.

[13] W.E. OLMSTEAD, Critical speed for the avoidance of blow-up in a reactivediffusive medium, Z. Angew. Math. Phys. 48 (1997), 701-710.

[14] V. PATA, G. PROUSE, M.I. VISHIK, Traveling waves of dissipative nonautonomous hyperbolic equations in a strip, Adv. Differential Equations 3 (1998), 249-270.

[15] V. PATA, A. ZUCCHI, Attractors for a damped hyperbolic equations with linear memory, submitted.

[16] R. TEMAM, Infinite-dimensional dynamical systems in mechanics and physics, Springer-Verlag, New York, 1988.

Received July 1999 